\title{
Paediatric urinary incontinence
}

\section{Gail Nankivell}

Senior physiotherapist ${ }^{1}$

Patrina HY Caldwell

Staff specialist

paediatrician ${ }^{1,2}$

1 The Children's Hospital

Westmead

${ }^{2}$ Discipline of Paediatrics and Child Health

University of Sydney

\section{Key words}

bedwetting, nocturnal

enuresis

Aust Prescr 2014,37:192-5

\section{SUMMARY}

Urinary incontinence, both in the day and at night, is common in school-aged children and can be very distressing for children and their families.

An accurate history together with a thorough physical examination is essential for assessing and diagnosing urinary incontinence.

Conservative treatment should be offered to all children. If that fails, treatment with anticholinergic drugs could be tried in those with daytime urinary incontinence and overactive bladder.

After addressing any daytime bladder symptoms, treatment with alarm therapy is recommended for children with nocturnal enuresis. Desmopressin is another option.

\section{Introduction}

Urinary incontinence in the day and at night is common in school-aged children. Its causes can be multifactorial. Daytime urinary incontinence occurs in about $17-20 \%$ of children ${ }^{1-3}$ with a further $6.6 \%$ of those having problems at night as well. ${ }^{2}$ The prevalence of nocturnal enuresis is $8-20 \%$ at five years of age, with a spontaneous remission rate of about $15 \%$ per year with persistence of enuresis in $0.5-2 \%$ of adults. ${ }^{4-6}$ Enuresis is more common in boys. Urinary incontinence can have a significant negative impact on a child's psychosocial well-being and quality of life.? Successful treatment can make a big difference. Assessment and management of these children should be based on recommendations of the International Children's Continence Society and the National Institute for Health and Clinical Excellence guidelines for nocturnal enuresis., ${ }^{8,9}$

\section{Definitions}

The terminology used to describe urinary incontinence is based on standards recommended by the International Children's Continence Society. ${ }^{8}$ Urinary incontinence is defined as involuntary wetting at an inappropriate time and place in a child aged five years or more. Continuous incontinence is usually associated with neurological or anatomical congenital malformations. Intermittent incontinence can occur during the day and at night.

\section{Physiology of bladder function}

The two functions of the bladder are to store and eliminate urine. With growth and maturity, day- and night-time continence is achieved in most children by about four years of age. ${ }^{10}$ However, it is normal for children to still have continence problems at this age.
During the day, voiding occurs when children synchronously contract their detrusor muscle and relax their urinary sphincters and pelvic floor muscles (usually in response to the sensation of bladder fullness). This allows the free flow of urine until the bladder is empty. At night, with adequate bladder storage and urine concentration, children usually sleep through the night without needing to urinate, but have the ability to wake up to void when they sense bladder fullness.

Bladder capacity increases with age in children. The normal expected bladder capacity up to the age of 12 is calculated as (age +1) $\times 30 \mathrm{~mL}$ (with $400 \mathrm{~mL}$ being expected for those older than 12 years). ${ }^{10}$ Normal daytime voided volumes are usually $65-150 \%$ of expected bladder capacity. These measures are the most useful indicator of bladder function.

Usually children produce less urine at night in response to the circadian variation in the amount of arginine vasopressin released by the pituitary gland." Nocturnal polyuria is overnight urine volumes greater than $130 \%$ of expected bladder capacity.

\section{Assessment}

An accurate history, including family history, together with a thorough physical examination is essential to evaluate children with urinary incontinence and decide on treatment choices. This includes a detailed history of wetting, toileting patterns including voiding frequency, symptoms of urgency, comorbidities and psychosocial factors. ${ }^{8}$

A bladder diary provides objective documentation of bladder function. Recording night-time urine volumes and incontinence episodes over seven nights is helpful to evaluate enuresis. Night-time urine volumes are estimated by adding the net nappy weight and the first morning voided volume. To evaluate daytime 
bladder function, a 48-hour chart can be used to measure frequency and volume of fluid intake and urine output during the day. Urine culture and uroflowmetry with abdominal ultrasound to assess post-void residual urine volumes and kidney anatomy can be helpful in children with daytime urinary symptoms, particularly when treatment has failed. Screening questionnaires for behavioural symptoms can help identify emotional and behavioural disorders which may need to be addressed.7,8

\section{Daytime urinary incontinence}

Children with daytime urinary incontinence may or may not also wet the bed at night. They often experience other lower urinary tract symptoms such as:

- $\quad$ urgency - the sudden and unexpected need to void

- urge incontinence - an inability to suppress voiding with urgency

- increased or decreased voiding frequency

- hesitancy

- $\quad$ straining

- a weak stream

- holding manoeuvres related to postponement of voiding - squatting or crossing legs.

Overactive bladder is a common disorder of urinary storage and commonly presents with urgency, frequency and urge incontinence. ${ }^{12}$ These children have small volume voids during the day. Children with infrequent voiding (less than four voids per day) may have voiding postponement. This may present with urgency and holding postures. Some of these children have behavioural problems. ${ }^{13,14}$ Dysfunctional voiding occurs when the child habitually contracts the external urethral sphincter during voiding. It is estimated to occur in $4-30 \%$ of children and is commonly associated with recurrent urinary tract infections. ${ }^{15}$ Risks for daytime urinary incontinence are listed in the Box. ${ }^{8,9,16}$

\section{Nocturnal enuresis}

Nocturnal enuresis without other lower urinary tract symptoms (monosymptomatic nocturnal enuresis) is more common than enuresis with lower urinary tract symptoms (non-monosymptomatic nocturnal enuresis). Enuresis is also classified as primary or secondary. Most children have primary enuresis and have never achieved night continence. Those who have previously been dry for at least six months have secondary enuresis. ${ }^{8}$

Enuresis involves poor sleep arousal in response to the sensation of a full bladder, abnormal bladder function or nocturnal polyuria. ${ }^{17}$ Known risk factors are listed in the Box. ${ }^{8,9,16}$ Constipation and overactive bladder are commonly associated with non- monosymptomatic nocturnal enuresis. Psychological comorbidities are more commonly associated with secondary enuresis. ${ }^{18}$

\section{Treatment}

Conservative therapy (also known as 'urotherapy') is non-surgical, non-pharmacological treatment for lower urinary tract symptoms and should be used in all children with urinary incontinence. It includes:

- education and advice about regular voiding and correct voiding posture

- avoiding holding manoeuvres

- encouraging adequate fluid intake

- managing constipation.

Managing constipation is important as there is a close relationship between bladder and bowel function. In one study, treatment of constipated children with a bowel program resolved daytime incontinence in $89 \%$ of cases, enuresis in $63 \%$ of cases and urinary tract infections in $100 \%$ of cases..$^{19}$ Sometimes conservative treatment alone can be effective. When it is inadequate for treating the child's urinary incontinence, other therapies could be tried.

\section{Alarm training}

Alarm training is the first-line therapy for nocturnal enuresis and the most effective long-term treatment. ${ }^{9,20}$ Enuresis alarms are wetness sensors

\section{Box Factors associated with urinary incontinence in children}

Daytime incontinence

- family history ${ }^{16}$

- abnormal bladder function

- vesicoureteral reflux

- uropathy (e.g. posterior urethral valves, epispadias)

- urinary tract infection

- constipation and faecal incontinence

- nocturnal enuresis

- neuropsychiatric or behavioural conditions such as attention deficit hyperactivity disorder, anxiety, depressive and conduct disorders

- intellectual disabilities ${ }^{8,9}$

Nocturnal enuresis

- family history ${ }^{16}$

- constipation

- faecal incontinence

- overactive bladder

- sleep apnoea and upper airway obstruction symptoms

- defective sleep arousal

- psychological factors 
which sound and wake the child when wet. They are either placed under the bed sheets ('bell and pad' or bed alarms) or worn in the child's underpants ('body worn' or personal alarms). Alarms work by training the child to hold on during sleep when they do not need to void, or to wake to void with a full bladder. ${ }^{20}$ Alarm training usually takes a few weeks to start working. It should be continued until 14 consecutive dry nights are achieved. Stopping earlier may result in relapse. Alarm training should be tried for a maximum of three months and can be used more than once. About two-thirds of children become dry during alarm training and nearly half remain dry after stopping. ${ }^{20}$ It is relatively inexpensive and potentially curative, but requires motivation and support as it can be quite difficult. ${ }^{18}$

\section{Desmopressin}

Desmopressin is considered a first-line drug therapy for enuresis. It is approved for treating nocturnal enuresis in children six years or older. Desmopressin works in about $70 \%$ of children, although less than half will become completely dry. ${ }^{21}$ It is a synthetic analogue of the pituitary hormone arginine vasopressin, which is an antidiuretic hormone. It reduces urine production overnight by increasing water reabsorption by the collecting tubules. Oral desmopressin (as a tablet or lyophilisate melt) has a lower risk for water intoxication than the nasal formulation. It is well tolerated and adverse effects such as headaches, abdominal pain and emotional disturbances are uncommon. As desmopressin can increase the risk for water intoxication and hyponatraemia, minimising fluid intake after taking the medication at night is essential. Desmopressin works best if nocturnal polyuria is present and daytime bladder function is normal. ${ }^{18}$ It is effective for short-term use when a rapid response is required or when alarm training is not suitable or effective. In therapy-resistant enuresis, it can be used in conjunction with other treatments. ${ }^{21}$ However, desmopressin has a high relapse rate, with only $18-38 \%$ of children remaining dry after stopping the drug. 22

\section{SELF-TEST}

QUESTIONS

True or false?

3. The expected bladder capacity of an eight-year-old child is $270 \mathrm{~mL}$.

4. Desmopressin is most effective for children with daytime incontinence.

Answers on page 219

\section{Anticholinergic drugs}

Anticholinergic drugs have an inhibitory effect on the detrusor muscle. They are thought to increase the capacity and compliance of the bladder and reduce unprovoked bladder contractions..$^{23,24}$ Children with overactive bladder, reduced bladder capacity and symptoms of urgency may benefit. Anticholinergics are commonly used for daytime incontinence associated with overactive bladder. for enuresis, it can improve a child's response when combined with other therapies (such as alarm or desmopressin) in treatment-resistant enuresis. ${ }^{25}$

Oxybutynin is the most commonly used anticholinergic. It is approved for use in children aged over five years who have overactive bladder. Adverse effects (such as decreased sweating and facial flushing, dry mouth, constipation, urinary hesitancy and retention and nervous system changes) are common and lead to treatment discontinuation in $10 \%$ of children. ${ }^{26}$ Newer bladder-specific anticholinergics - such as tolterodine and solifenacin - are increasingly being used in children as they have fewer adverse effects. However, evidence supporting paediatric use is limited. Anticholinergic drugs can cause constipation and incomplete bladder emptying so it is important to monitor for this.

\section{Tricyclic antidepressants}

Imipramine can be used to treat enuresis in children aged over six years. It is moderately effective, with about one fifth becoming dry on treatment, but most relapse when treatment is ceased. ${ }^{27}$ Most children tolerate tricyclics without experiencing adverse effects, with the main complaints being dry mouth, gastrointestinal symptoms and behavioural changes. However, due to the risk of potentially serious adverse effects (cardiac arrhythmias, hypotension, hepatotoxicity, central nervous system depression and interaction with other drugs) and the danger of overdose, tricyclics require close supervision and should be stored in a secure location. They are used only in therapy-resistant enuresis.

\section{Referral}

Referral to specialist services should be considered for children with severe daytime symptoms, recurrent urinary tract infections, physical or neurological problems and psychosocial or other comorbidities requiring further management. Referral should also be considered when treatment is not successful after six months.

\section{Conclusion} Although anticholinergic monotherapy is ineffective
Daytime urinary incontinence and nocturnal enuresis are common in children, and are frequently encountered in general practice. This stigmatising condition can have a significant impact on a child and their family, and successful treatment can be life-changing. A thorough history and examination is essential for an accurate diagnosis and for determining the best treatment. $<$

Conflict of interest: none declared 


\section{REFERENCES}

The full list of references and further reading is published with the online version of this article at www.australianprescriber.com/magazine/37/6/192/5

8. Austin PF, Bauer SB, Bower W, Chase J, Franco I, Hoebeke P, et al. The standardization of terminology of lower urinary tract function in children and adolescents: update report from the Standardization Committee of the International Children's Continence Society. J Urol 2014;191:1863-5.
9. National Institute for Health and Care Excellence. Nocturnal enuresis: the management of bedwetting in children and young people. NICE Guidelines [CG111]. 2010 www.nice.org.uk/guidance/cg111 [cited 2014 Nov 3]

\section{Top 10 drugs}

\section{Table 1 Top 10 drugs by DDD/1000 pop/day *+}

\begin{tabular}{llr}
\hline Constituent drug & PBS/RPBS $\ddagger$ \\
\hline 1. & atorvastatin & 69.31 \\
2. & rosuvastatin & 41.87 \\
3. & paracetamol & 33.65 \\
4. & perindopril & 32.73 \\
5. & irbesartan & 28.49 \\
6. & amlodipine & 26.73 \\
7. & esomeprazole & 24.90 \\
8. & candesartan & 23.38 \\
9. & ramipril & 22.43 \\
10. & simvastatin & 17.94 \\
\hline
\end{tabular}

\section{Table 2 Top 10 drugs by prescription counts ${ }^{\dagger}$}

\begin{tabular}{llc}
\hline Drug & PBS/RPBS $\ddagger$ \\
\hline 1. & atorvastatin & 8659127 \\
2. rosuvastatin & 7610977 \\
3. esomeprazole & 6967801 \\
4. paracetamol & 6299698 \\
5. pantoprazole & 4179926 \\
6. perindopril & 3951282 \\
7. metformin hydrochloride & 3539513 \\
8. salmeterol and fluticasone & 3168825 \\
9. irbesartan & 3032109 \\
10. simvastatin & 2974311 \\
\hline
\end{tabular}

\section{Table 3 Top 10 drugs by cost to government ${ }^{+}$}

\begin{tabular}{|c|c|c|c|c|}
\hline \multicolumn{2}{|c|}{ Drug } & \multirow{2}{*}{$\begin{array}{r}\text { Cost to government } \\
\text { (A\$) }\end{array}$} & \multirow{2}{*}{$\begin{array}{r}\begin{array}{r}\text { DDD/1000 pop/day * } \\
\text { PBS/RPBS } \ddagger\end{array} \\
41.87\end{array}$} & \multirow{2}{*}{$\begin{array}{c}\begin{array}{c}\text { Prescriptions } \\
\text { PBS/RPBS }\end{array} \\
7610977\end{array}$} \\
\hline 1. & rosuvastatin & & & \\
\hline 2. & adalimumab & 270237753 & 0.50 & 152563 \\
\hline 3. & atorvastatin & 259067466 & 69.31 & 8659127 \\
\hline 4. & esomeprazole & 204253775 & 24.90 & 6967801 \\
\hline 5. & etanercept & 153005371 & 0.29 & 87283 \\
\hline 6. & insulin glargine & 131811872 & 6.94 & 320806 \\
\hline 7. & tiotropium bromide & 131776620 & 7.03 & 1993769 \\
\hline 8. & fingolimod & 113531100 & 0.16 & 49576 \\
\hline 9. & olanzapine & 109682018 & 3.09 & 993826 \\
\hline & quetiapine & 99773937 & 2.62 & 978353 \\
\hline
\end{tabular}

* The defined daily dose (DDD)/thousand population/day is a more useful measure of drug utilisation than prescription counts. It shows how many people in every thousand Australians are taking the standard dose of a drug every day. DDD includes use in combination products.

+ Based on date of supply. Does not include private prescriptions or prescriptions under PBS co-payment.

‡ PBS Pharmaceutical Benefits Scheme, RPBS Repatriation Pharmaceutical Benefits Scheme

Source: Department of Health, 10 October 2014. (c) Commonwealth of Australia.

The cost figures exclude any patient contributions. 